\title{
Czochralski Method for Crystal Growth of Reactive Intermetallics
}

\author{
E. TALIK* AND M. OBOZ \\ Institute of Physics, University of Silesia, Uniwersytecka 4, 40-007 Katowice, Poland
}

\begin{abstract}
Czochralski method of crystallization allows obtaining single crystals of many intermetallic compounds. New series $\mathrm{Gd}_{7} \mathrm{Ni}_{x} \mathrm{Pd}_{3-x}(x=2,3)$ intermetallics was grown by the modified Czochralski method using a levitated melt in protective argon atmosphere. Preliminary studies of magnetic properties have shown that all crystals of the series order ferromagnetically. It was found that $\mathrm{Gd}_{7} \mathrm{Ni}_{2} \mathrm{Pd}$ shows magnetic transition to the ferromagnetic state at $225 \mathrm{~K}$ while $\mathrm{Gd}_{7} \mathrm{Ni}_{3}$ orders at $145 \mathrm{~K}$.
\end{abstract}

DOI: 10.12693/APhysPolA.124.340

PACS: 71.20.Lp, 81.10.Fq, 61.66.Dk

\section{Introduction}

Method of crystallization worked out by Czochralski in 1916 proves to be very useful for crystallization of many intermetallics. Czochralski invented this simple method for crystal growth during the experiments measuring the crystallization velocity of metals. The idea of this method was based on pulling of fibers of different metals from their melts. The obtained in such way metallic wires proved to be single crystals. The results of the experiments Czochralski published in Zeitschrift für Physikalische Chemie in 1918 [1]. This new technique allowed him to obtain the good quality single crystals of pure metals like $\mathrm{Sn}, \mathrm{Pb}, \mathrm{Zn}$ grown in air.

Later this method was adopted for crystal growth of semiconductors and oxides for electronic application. Moreover, a modified Czochralski method allowed to obtain the single crystal of the intermetallic compounds, containing components even very reactive, sensitive to oxygen and other contamination. After separation of the growing crystal from air, by use of growth chamber, an application of the protective atmosphere of inert gas e.g. argon was possible. The application of the chamber additionally allows to heat starting materials under dynamic vacuum to remove adsorbed gases and additionally to control the gas pressure during growth process. The next source of a possible contamination of the crystal is a contact of the melt with material of crucible. To eliminate this problem a water cooled cold crucible or levitating coil, divided into segments with a proper profile, can be used [2]. The levitation of ingot additionally allows to avoid the transport of the heat from the melt to the crucible and thus melting the materials even with high melting point. For investigation of the compounds containing the expensive noble elements a reduction of the cost of starting materials is available by decrease of the ingot mass. In our modified Czochralski device it is possible to

\footnotetext{
*corresponding author; e-mail: talik@us.edu.pl
}

pull out the single crystals from a small drop of the melt (mass of starting materials from $1 \mathrm{~g}$ to several grams). The obtained crystals were $\approx 2 \mathrm{~mm}$ in diameter and several $\mathrm{cm}$ in length. The size of the crystals grown using small ingot is enough for the basic investigations.

However, there are a limitation of the Czochralski method to the growth from an ingot which melt congruently (see below). There are several different methods of the crystal growth of intermetallics described in literature as e.g. flux growth, floating-zone melting, Bridgman growths, Tri-Arc growths etc. [3-6]. But often the mass of the ingot is several times larger than described above in our device.

\section{Advantages and disadvantages of Czochralski method}

The Czochralski method possesses the several features as:

- extraction of the crystal in the opposite direction to the gravitation,

- crystallized materials are withdrawn from an ingot which melt congruently (without decomposition into the different phases). However, if the separation between the temperature of liquidus and peritectic line is narrow in the phase diagram, crystallization of the required compound is observed [7];

- spontaneous nucleation or by use of the oriented nucleus. Czochralski immersed capillary in the melt for spontaneous nucleation of a single crystal. However, the spontaneous nucleation is also possible by necking of the withdrawn crystal in order to isolate single grain. This grain would expand into single crystal during the growth process;

- free growth, without tension caused by the limitations of the crucible;

- possibility of applying the protective atmosphere; 
- comparatively quick process of receiving the crystals and the possibility of receiving large crystals important in industrial growth processes. Possible weight control of the growing crystal.

Currently used techniques allow to control operating parameters, such as:

- heating power and temperature of ingot leading to changes e.g. viscosity, surface tension. Such changes influence the diameter of the crystal and allow to increase the mass of crystal or finish the growth process;

- speed of the extraction;

- rotation, which influences the field of the temperature and the field of the concentration of components and shape of front of crystallization;

- use magnetic fields for suppression of the convection. This is important to obtain good quality (less defected) crystals for industrial application;

- possibility of re-melting the grown material.

The control of growth parameters can be related to modeling and development of the theory of crystal growth.

To disadvantages of the method belong:

- tensions during the cooling down, necessity of warming up of the grown crystal, especially large;

- possible segregation of constituents.

\section{Czochralski method for intermetallics crystal growth}

Prospective applications of $\mathrm{R}-\mathrm{T}(\mathrm{R}=$ rare earth, $\mathrm{T}=$ transition metal) intermetallics are following: magnetic refrigeration where the magnetic materials with relatively high magnetic transition temperatures are desired; spintronics where materials with layered-like or atomic-chain structures show a large reduction of the electrical resistivity under magnetic field, called the giant magnetoresistivity; hydrogen storage, strong magnets; materials science, etc.

Understanding of the nature of interactions in solid state and anisotropy of properties are desired for applications of new materials. Such credible measurements results can be realized using good quality single crystals for investigations of e.g. the temperature dependence of lattice parameters, magnetic and electrical properties along principal directions. Moreover, crystal growth of the intermetallic compounds is important in order to: eliminate effects of grain boundaries, essential for transport and magnetic properties examinations; measure anisotropy of properties of new materials; examine the electronic structure, where cleanness of material is essential, etc. The examples of such measurements are the X-ray photoelectron spectroscopy (XPS) electronic structure examinations of the $\mathrm{RMn}_{2}(\mathrm{R}=$ rare earth) or actinides intermetallics [8, 9]. Because these materials are extremely reactive, so only compact monocrystalline samples broken under UHV condition, enable obtaining of fresh and clean surface and credible results. Even small oxidation of the surface gives a pronounced disturbance of the valence band or uranium $4 f$ states. The XPS spectra only from clean surface inform about localized or itinerant character of the electrons around the Fermi level and allow a proper comparison with the electronic structure calculations.

Recently magnetic materials having relatively high Curie temperatures $\left(T_{\mathrm{C}}\right)$ are widely investigated as potential candidates for magnetic refrigeration. They should have a large magnetocaloric effect (MCE). The examination of the crystal and electronic structure, magnetic and electrical properties of the new magnetic materials single crystals $\mathrm{Gd}_{7-x} \mathrm{Y}_{x} \mathrm{Pd}_{3}(x=0-6)$ were performed $[10-15]$. They all crystallize in the $\mathrm{Th}_{7} \mathrm{Fe}_{3}$-type structure. In $\mathrm{Gd}_{7-x} \mathrm{Y}_{x} \mathrm{Pd}_{3}$ compounds $\mathrm{Gd}$ ions in their magnetic sublattice possess a large and localized magnetic moment. The exchange interactions between $\mathrm{Gd}$ ions are a dominating mechanism being responsible for the ferromagnetic ordering. This mechanism gradually decreases with the growing non-magnetic yttrium content. However, besides of above mentioned exchange interactions the other contributions have to be considered. In the unit cell of the $\mathrm{Th}_{7} \mathrm{Fe}_{3}$-type structure, the $\mathrm{Gd}$ ions are located in the triangular configuration. Therefore, a magnetic frustration as an additional factor must be taken into account. The Gd substitution by the yttrium atoms destroys partly the triangular arrangement of the Gd moments. Thus the effect of the magnetic frustration weakens with increase of the $\mathrm{Y}$ content. The next mechanism responsible for magnetic properties of $\mathrm{Gd}_{7-x} \mathrm{Y}_{x} \mathrm{Pd}_{3}$ is the itinerant magnetism originating from the $d$-bands around the Fermi level due to hybridization of Gd $5 d, \mathrm{Y} 4 d$ and $\mathrm{Pd} 4 d$. With increasing $\mathrm{Y}$ content the ferromagnetic transitions shift down to lower temperatures from $334 \mathrm{~K}$ for $\mathrm{Gd}_{7} \mathrm{Pd}_{3}$ to $75 \mathrm{~K}$ for $\mathrm{GdY}_{6} \mathrm{Pd}_{3}$. So yttrium substitution allows tuning up the transition temperature [15].

To eliminate an expensive $\mathrm{Pd}$ metal and to examine the influence of the transitions metals on the magnetic interactions and magnetocaloric effect, the substitution of $\mathrm{Pd}$ by $\mathrm{Ni}$ in a series of new magnets for magnetic refrigeration was realized. As the first there was examined the single crystal of $\mathrm{Gd}_{7} \mathrm{NiPd}_{2}[16] . \mathrm{Gd}_{7} \mathrm{NiPd}_{2}$ undergoes a long-range ferromagnetic-type ordering at $T_{\mathrm{C}}=298 \mathrm{~K}$, slightly higher than elemental Gd (293 K), followed by an antiferromagnetic spin-reorientation below $135 \mathrm{~K}$. The magnetization data indicate that there is an excess magnetic moment calculated per $\mathrm{Gd}^{3+}$ ions coming from the hybridized $d$ bands. The measured XPS valence band shows the hybridization effect between Gd $5 d$, Ni $3 d$ and $\mathrm{Pd} 4 d$ states. The calculated values of the entropy $\Delta S_{\mathrm{m}}$ 
for the $\mathrm{Gd}_{7} \mathrm{NiPd}_{2}$ compound is $-6.85 \mathrm{~J} /(\mathrm{kg} \mathrm{K})$ for the $a$ axis and -6.49 for the $c$ axis at $7 \mathrm{~T} \mathrm{[16]} \mathrm{and} \mathrm{is} \mathrm{not}$ lower than for the parent $\mathrm{Gd}_{7} \mathrm{Pd}_{3}(6.67 \mathrm{~J} /(\mathrm{kg} \mathrm{K})$ for $a$ direction and $6.68 \mathrm{~J} /(\mathrm{kg} \mathrm{K})$ for $c$ direction $)$.

\section{Experimental}

The crystals of $\mathrm{Gd}_{7} \mathrm{Ni}_{x} \mathrm{Pd}_{3-x}(x=2,3)$ were grown from a small amount (about $1 \mathrm{~g}$ ) of the starting materials. The starting materials Gd (99.9\% purity), Ni, Pd (Specpure Johnson Matthey Chemical Ltd.) which were melted together on a water cooled eight-segment conical coil with a diameter of $14 \mathrm{~mm}$ in stoichiometric amounts of elements. They were melted together under very pure argon atmosphere in a Czochralski-type apparatus [2]. The obtained ingots were remelted several times to get good homogenization. The crystal growth process was started by immersion of a molybdenium spike in the melt. The strong stirring of the melt ensured homogenization of the sample during the growth process. After necking, a crystal was pulled out with a constant velocity of $0.1 \mathrm{~mm} / \mathrm{min}$. The obtained crystals were cylindrical with a diameter of about $3 \mathrm{~mm}$ and a length of $20 \mathrm{~mm}$. The photos of the $\mathrm{Gd}_{7} \mathrm{Ni}_{x} \mathrm{Pd}_{3-x}(x=2,3)$ crystals are shown in Figs. 1 and 2. The single crystal X-ray diffraction was performed at room temperature on a four-circle KM4 diffractometer using graphite-monochromatized Mo $K_{\alpha}$ radiation. The intensities of reflections were corrected for the Lorentz and polarization effects. Magnetization was measured using a Quantum Design MPMSXL-7AC SQUID magnetometer in the temperature range $2-400 \mathrm{~K}$ in the magnetic field $0.1 \mathrm{~T}$.

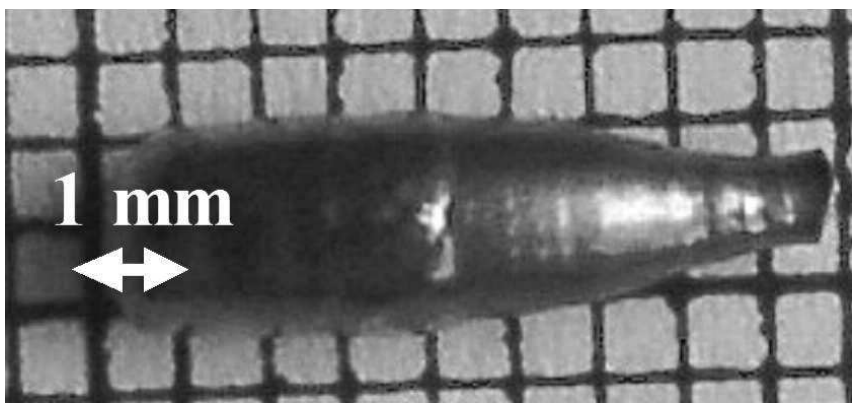

Fig. 1. A view of $\mathrm{a}_{7} \mathrm{Ni}_{2} \mathrm{Pd}$.

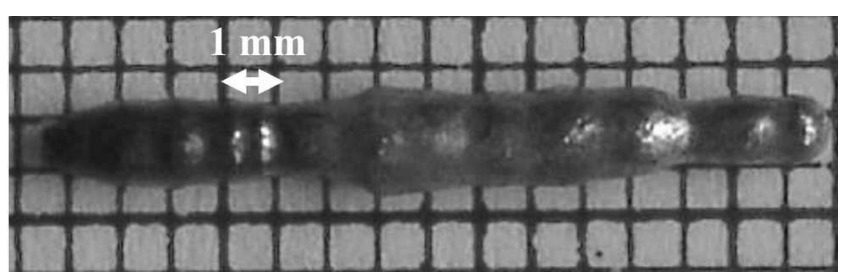

Fig. 2. A view of $\mathrm{a}_{\mathrm{Gd}} \mathrm{Ni}_{3}$.

\section{Results and discussion}

The obtained $\mathrm{Gd}_{7} \mathrm{Ni}_{2} \mathrm{Pd}$ crystallized, as the parent compound $\mathrm{Gd}_{7} \mathrm{Pd}_{3}$, in $\mathrm{Th}_{7} \mathrm{Fe}_{3}$-type of hexagonal crystal structure with a space group $P 6_{3} m c$. The lattice parameters of the parent compound $\mathrm{Gd}_{7} \mathrm{Pd}_{3}$ are $a=9.981(1) \AA, c=6.279(1) \AA$ and unit cell volume $541.7(2) \AA^{3}$. For the compound $\mathrm{Gd}_{7} \mathrm{NiPd}_{2}$ the lattice parameters are $a=9.372(5) \AA, c=6.2674(4) \AA$ and unit cell volume $536.0(1) \AA^{3}[16]$. The lattice parameters for the compound with $\mathrm{Gd}_{7} \mathrm{Ni}_{2} \mathrm{Pd}$ are $a=9.8504(8) \AA$, $c=6.228(6) \AA$ and unit cell volume $523.40(8) \AA^{3}$. A decrease of the lattice parameters indicate the substitution palladium by nickel. Figure 3 shows the unit cell volume decrease with growing $x$ for both series. In spite of that the metallic radius of $\mathrm{Y}(1.80 \AA)$ is slightly higher than $\operatorname{Gd}(1.789 \AA)$, the decrease of the unit cell volume occurs due to hybridization process. For Pd such radius is $1.37 \AA$, while for $\mathrm{Ni}$ is smaller and equals $1.24 \AA$ so the decrease is more rapid. The $\mathrm{X}$-ray diffraction (XRD) spectrum of the sample $\mathrm{Gd}_{7} \mathrm{Ni}_{3}$ showed besides the main phase some extra contributions. In order to obtain one phase sample the thermal treatment is necessary.

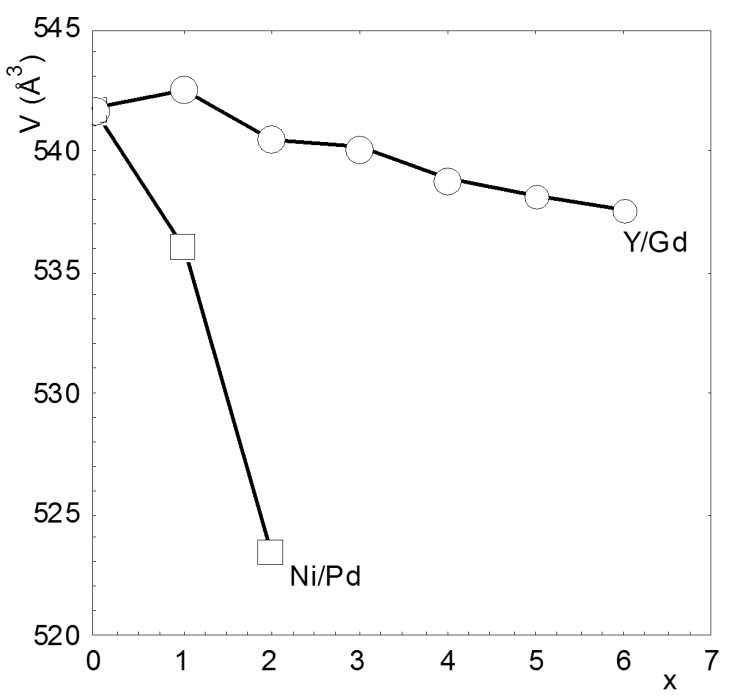

Fig. 3. Unit cell volume of $\mathrm{Gd}_{7-x} \mathrm{Y}_{x} \mathrm{Pd}_{3}$ and $\mathrm{Gd}_{7} \mathrm{Ni}_{x} \mathrm{Pd}_{3-x}$ series.

Preliminary investigations for the non-oriented crystals of the magnetic moment temperature dependence for $\mathrm{Gd}_{7} \mathrm{Ni}_{x} \mathrm{Pd}_{3-x}$ were carried out. In Fig. 4 the temperature dependences of the magnetization show the transitions to the ferromagnetic state at $298 \mathrm{~K}$ for $\mathrm{Gd}_{7} \mathrm{NiPd}_{2}$, $225 \mathrm{~K}$ for $\mathrm{Gd}_{7} \mathrm{Ni}_{2} \mathrm{Pd}$ and at $145 \mathrm{~K}$ for $\mathrm{Gd}_{7} \mathrm{Ni}_{3}$ compounds. The dependence of the ordering temperature versus nickel and yttrium content for both series is shown in Fig. 5. The literature data for the amorphous ferromagnet $\mathrm{Gd}_{7} \mathrm{Ni}_{3}$ show the ordering temperature $120 \mathrm{~K}$ and a blur magnetization run [17].

Substitution of the non-magnetic yttrium into gadolinium sublattice leads to new mechanisms of interac- 


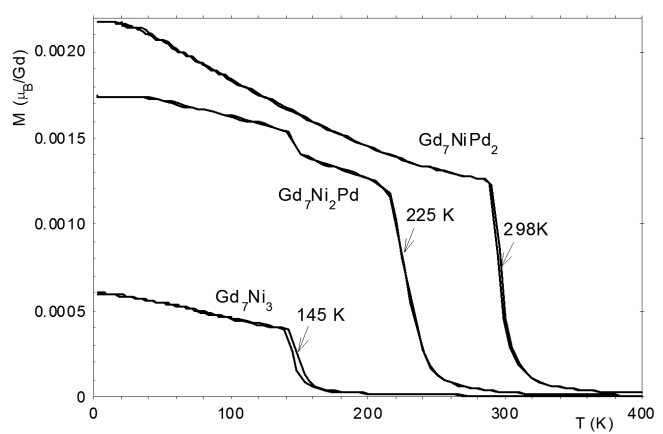

Fig. 4. Magnetization of $\mathrm{Gd}_{7} \mathrm{Ni}_{x} \mathrm{Pd}_{3-x}$ series.

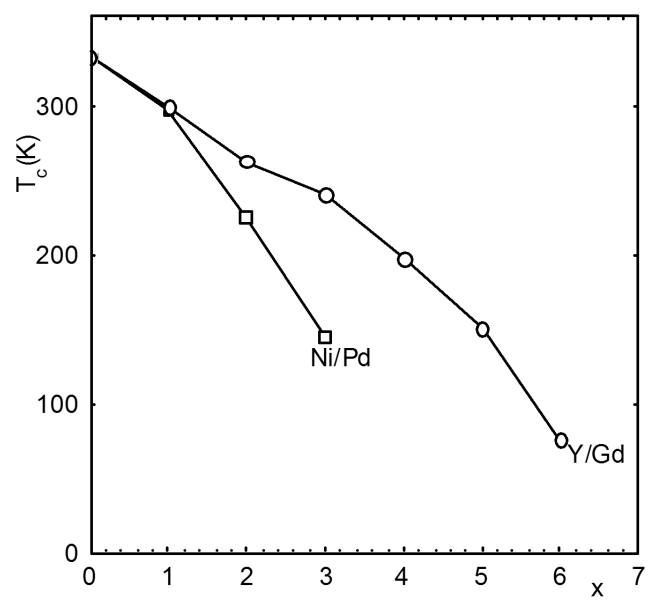

Fig. 5. The Curie temperatures along the $\mathrm{Gd}_{7-x} \mathrm{Y}_{x} \mathrm{Pd}_{3}$ and $\mathrm{Gd}_{7} \mathrm{Ni}_{x} \mathrm{Pd}_{3-x}$ series.

tions: the modification of the exchange RudermanKittel-Kasuya-Yosida (RKKY) interactions between Gd ions (decrease of ordering temperature), the hybridization process via Y $4 d$ (enhanced magnetic coupling [18]), magnetic disorder (transport and magnetic properties), decrease of the magnetic frustration (transport and magnetic properties). Substitution of nickel into palladium sublattice in $\mathrm{Gd}_{7} \mathrm{Ni}_{x} \mathrm{Pd}_{3-x}$ series changes the hybridization mechanism (now via Ni $3 d$ states) which influences in different way on the magnetic interactions and magnetocaloric properties which will be the object of further examinations.

\section{Summary}

As for the recently examined series $\mathrm{Gd}_{7-x} \mathrm{Y}_{x} \mathrm{Pd}_{3}$, the decrease of the transition temperature is observed for the preliminary examined $\mathrm{Gd}_{7} \mathrm{Ni}_{x} \mathrm{Pd}_{3-x}$ series together with maintenance of the type of crystal structure for $x=1,2$. The observed decrease of the unit cell volume and or- dering temperature is more rapid for the nickel substituted compounds. The obtained new series of ferromagnetic materials will be checked not only in relation to the change of the magnetic entropy and magnetocaloric efficiency but magnetic interaction as well. Such materials could find application in the multistage magnetic refrigerators covering the temperature range down to $145 \mathrm{~K}$.

\section{Acknowledgments}

This work was financed by the Polish National Science Centre (Narodowe Centrum Nauki) under grant no. 2011/03/B/ST5/01035.

\section{References}

[1] J. Czochralski, Z. Phys. Chem. 92, 219 (1918).

[2] E. Talik, J. Szade, J. Heimann, A. Winiarska, A. Winiarski, A. Chelkowski, J. Less-Common Met. 138, 129 (1988).

[3] S. Sarkar, S.C. Pete, J. Chem. Sci. 124, 1385 (2012).

[4] Ch. Cao, W. Löser, G. Behr, R. Klingeler, N. Leps, H. Vinzelberg, B. Büchner, J. Cryst. Growth 318, 1009 (2011).

[5] A. Onyszko, W. Bogdanowicz, K. Kubiak, J. Sieniawski, Cryst. Res. Technol. 45, 1326 (2010).

[6] D. Fort, Rev. Sci. Instrum. 68, 3504 (1997).

[7] E. Talik, M. Klimczak, A. Winiarski, R. Troc, J. Cryst. Growth 283, 547 (2005).

[8] E. Talik, M.E. Lucas, W. Suski, R. Troć, J. Alloys Comp. 350, 72 (2003).

[9] E. Talik, M. Kulpa, J. Alloys Comp. 286, 158 (1999).

[10] E. Talik, M. Klimczak, R. Troć, J. Kusz, W. Hofmeister, A. Damm, J. Alloys Comp. 427, 30 (2007).

[11] E. Talik, M. Klimczak, R. Troć, J. Kusz, W. Hofmeister, A. Winiarski, J. Alloys Comp. 460, 1 (2008).

[12] E. Talik, M. Klimczak, A. Winiarski, R. Troć, J. Cryst. Growth 310, 1886 (2008).

[13] E. Talik, M. Klimczak, V.H. Tran, J. Kusz, W. Hofmeister, A. Winiarski, R. Troć, Intermetallics 10, 27 (2010).

[14] E. Talik, M. Oboz, V.H. Tran, J. Kusz, W. Hofmeister, A. Winiarski, J. Cryst. Growth 312, 1651 (2010).

[15] E. Talik, M. Oboz, J. Kusz, A. Winiarski, W. Hofmeister, J. Alloys Comp., submitted for publication.

[16] M. Oboz, E. Talik, J. Kusz, A. Winiarski, J. Alloys Comp. 509, 4478 (2011).

[17] I. Nakai, T. Fukagawa, J. Phys. Soc. Jpn. 62, 2456 (1993).

[18] A. Woźniakowski, J. Deniszczyk, W. Borgieł, Acta Phys. Pol. A 121, 1060 (2012). 